\title{
Yellow Feet in a Patient with Breast and Thyroid Carcinoma, Due to Oral Intake of Turmeric
}

\author{
Liran Horev $\cdot$ Yuval Ramot $\cdot$ Laurent Klapholz
}

Published online: 26 March 2015

(C) The Author(s) 2015. This article is published with open access at Springerlink.com

\begin{abstract}
Yellow discoloration of the skin may be caused by several etiologies, including jaundice, hypervitaminosis, drug reaction or chemical exposure. Herein we describe a 68-year-old woman with a history of breast and thyroid carcinoma, presenting with a yellow discoloration of her soles, after ingestion of one capsule a day of turmeric root extract (Bluebonnet Turmeric Root, $500 \mathrm{mg}$, Vcap), taken for its anti-cancer properties, for 4 months. After drug cessation, the yellow hue disappeared completely. A Naranjo assessment score of 5 was obtained, indicating a probable relationship between the patient's symptom and her use of the drug. Curcumin, a mixture of diferuloymethane derivatives known as curcuminoids, is a yellow pigment present in the spice turmeric. Topical application of curcumin to the human skin is joined by orange-yellow discoloration. To the best of our knowledge, yellow skin discoloration after oral intake of turmeric is not mentioned in the medical literature.
\end{abstract}

L. Horev $(\bowtie) \cdot$ Y. Ramot · L. Klapholz

Department of Dermatology, Hadassah-Hebrew University

Medical Center, PO Box 12000, 9112001 Jerusalem, Israel

e-mail: liran.horev@gmail.com

\section{Key Points}

Turmeric root extract contains curcuminoids, a mixture of diferuloymethane derivatives that gives it its yellow pigment.

Topical application of curcuminoids to the human skin is joined by orange-yellow discoloration.

We describe a patient in which a yellow discoloration of the soles is attributed to the oral consumption of a $500 \mathrm{mg}$ capsule of turmeric root extract daily, for 4 months. A Naranjo assessment score of 5 indicates a probable relationship between the patient's symptom and her use of the drug.

\section{Introduction}

Skin discoloration is infrequently observed in the setting of a dermatology clinic. It may be generalized or localized and may have different hues such as yellow, grey or bluish. Differential diagnosis may be wide. Herein we describe a patient with breast and thyroid carcinoma, presenting with yellow discoloration of her soles due to oral ingestion of turmeric (curcumin).

\section{Case Report}

A 68-year-old woman presented to the dermatology outpatient clinic with a 3-month history of yellow discoloration of her soles. Ten years earlier, a breast carcinoma was diagnosed, and she was treated surgically and with 
chemotherapy. Three years prior to presentation, a second primary carcinoma of the thyroid gland was diagnosed and the patient underwent a second operation.

At the time of presentation, her medicaments included exemestane (Aromasin), an aromatase inhibitor, an anticancer drug that blocks androgen conversion to estrogen, and levothyroxine (Eltroxin). She also took a wide variety of vitamins and dietary supplements, for a prolonged time, including $\mathrm{Ca}^{2+} / \mathrm{Mg}^{2+}$ citrate, vitamin $\mathrm{D}$, multi-vitamin, Lglutamate, omega 3, Ganoderma lucidum (Ling zhi) mushroom, Polygonatum odoratum ( $P$. officinale) and white mulberry (Morus alba), the latter three prescribed by her naturopathic doctor. Turmeric root extract, (Bluebonnet Turmeric Root, 500 mg, Vcap) was started by her naturopath 4 months before presentation, once a day. Bluebonnet Turmeric Root Extract Vcaps contain $95 \%$ curcuminoids: curcumin $332 \mathrm{mg}$, demethoxycurcumin $71 \mathrm{mg}$, bisdemethoxycurcumin $11 \mathrm{mg}$, turmeric root powder (Curcuma C3 Complex ${ }^{\circledR}$, Curcuma longa) $150 \mathrm{mg}$. Other ingredients are from the vegetable capsules, Fruit02X ${ }^{\circledR}$ : wild blueberry, cranberry, prune, cherry and bilberry fruits, grape seed, raspberry seed, vegetable cellulose, calcium phosphate, silica, and vegetable magnesium stearate. The patient denied administration of any topical treatment to her feet or any change in her diet.

At physical examination, both feet and toenails showed a bright yellow discoloration, with hyperkeratotic areas with a more pronounced pigmentation (Fig. 1). No color change was present on the palms, conjunctivae or oral mucosae and tongue. Laboratory work up disclosed the presence of anemia, with normal liver, kidney and thyroid function tests, lipids and vitamin A levels. CA-125 (cancer

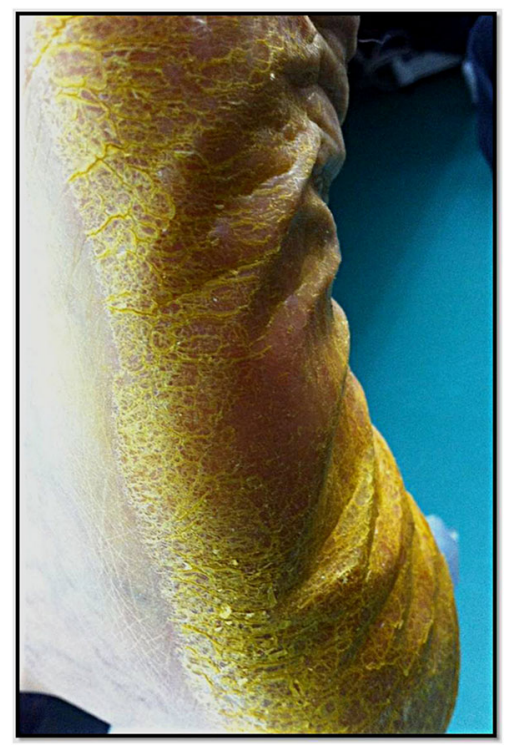

Fig. 1 Yellow discoloration of the sole, more pronounced in areas of hyperkeratotic skin antigen 125) and CA-15.3 (cancer antigen 15.3) were mildly elevated.

The patient was advised to stop taking the turmeric root extract. Two months later the skin discoloration disappeared completely.

\section{Discussion}

Yellow discoloration of the skin may be caused by several etiologies, including jaundice, hypervitaminosis, drug reaction or chemical exposure. In our case, jaundice was suspected, because the patient has a history of carcinomas, and could have jaundice due to intra-hepatic or post-hepatic metastatic disease. Other possible etiologies include hemolysis, alcohol ingestion, infectious hepatitis, drug reaction, autoimmunity, gallstones and biliary tract infection. However, all different types of jaundice are characterized by yellow conjunctivae and elevated bilirubin [1].

Although our patient had elevated cancer markers, liver function tests were normal, and no involvement of the conjunctivae was evident. The lack of hyperbilirubinemia excluded completely this diagnosis.

'Carotenoderma' is the name given to the yellow discoloration of the skin that is caused by increased serum carotenoids. Carotenoids are absorbed by passive diffusion from the gastrointestinal tract and are then partially metabolized in the intestinal mucosa and liver to retinol (vi$\operatorname{tamin}$ A). From there they are transported in the plasma via lipoproteins into the peripheral tissues, and in the skin they are deposited in the intercellular lipids of the stratum corneum. Therefore, color change is most prominent in regions of increased sweating and thickness of this layer. These areas include the palms, soles, knees, and nasolabial folds. The primary factor differentiating carotenoderma from jaundice clinically is the characteristic sparing of the conjunctivae in carotenoderma. Carotenoderma can be divided into primary and secondary carotenoderma. Primary carotenoderma develops from increased oral ingestion of foods such as carrots, tomatoes and squash, whereas secondary carotenoderma is caused by underlying diseases that increase serum carotenoids with normal oral intake, such as hypothyroidism, diabetes mellitus, anorexia nervosa, nephrotic syndrome and liver disease. Some of these diseases have in common an increased level of lipids and lipoproteins in the serum. Serum vitamin A would be elevated in all these cases, but was normal in our case, excluding this diagnosis [2].

Yellow skin discoloration was reported with the use of several medications, including the multitargeted tyrosine kinase inhibitors sorafenib and sunitinib [3], trastuzumab [4], quinacrine and dipyridamole. Canthaxanthin, a carotenoid, is authorized in some countries for trout, salmon 
and poultry feed and is associated with skin yellowing with touch. Canthaxanthin has also been used orally as an artificial tanner because of the orange-tan discoloration it applies to the skin, but was associated with health problems and banned. None of the above was taken by our patient.

Among chemical exposures, dinitrophenol or its derivative, picric acid, methylenedianiline and saffron (Crocus sativus), are all associated with yellowing of the skin, hair, and nails at sites of contact [5]. The patient did not report exposure to any of these agents.

Curcumin, a mixture of diferuloymethane derivatives known as curcuminoids, is a yellow pigment present in the spice turmeric (Curcuma longa, C. domestica, Indian saffron), which gives curry powder its color and flavor. It is added to sunscreens and cosmetics due to its anti-oxidative properties and is used as food colorant in butter and cheese. Turmeric has been used in traditional medicine for centuries, thought to harbor anti-oxidant, anti-inflammatory, anti-cancer, anti-viral, and anti-bacterial qualities. There are more than 2400 references in PubMed about turmeric as a cancer suppressor [6]. Curcuminoids are not harmful in high doses. According to the assessment report on C. longa of the committee on herbal medicinal products of the European Medicines Agency, only minor side effects such as dry mouth, flatulence and gastric irritation are expected in clinical use, as no serious side effects have been described [7]. Curcumin has a poor bioavailability and solubility. The major portion of ingested curcumin is excreted through the feces unmetabolized. However, the small portion that is absorbed is conjugated to glucuronides and sulfates, in the gut, via UDP-glucuronosyltransferase (UGT) and sulfotransferase (SULT) enzymes $[8,9]$. These water-soluble metabolites can be delivered to tissues. It seems possible that curcuminoids deposit in the intercellular lipids of the stratum corneum in a similar manner to the carotenoids, explaining the predilection of yellowing to thicker skin areas. Topical application of curcumin to the human skin is considered beneficial in several skin diseases but is joined by bright orange-yellow discoloration [10].

Clinical studies did not report skin yellowing as a side effect of oral consumption of high doses of curcumin or of curcumin with better bioavailability [11]. To the best of our knowledge, although known among naturopathic practitioners, this is the first report of yellow skin discoloration after oral intake of turmeric in the medical literature. Since the use of turmeric in non-conventional medicine is growing, it is important for dermatologists and other practitioners to become familiar with its yellow discoloration effects.

Acknowledgements Drs. Liran Horev, Yuval Ramot and Laurent Klapholz declare that they have no conflict of interest. No financial support was received for the conduct of this study or preparation of this manuscript.

Informed consent The patient gave a written informed consent to the publication of this case report.

Open Access This article is distributed under the terms of the Creative Commons Attribution Noncommercial License which permits any noncommercial use, distribution, and reproduction in any medium, provided the original author(s) and the source are credited.

\section{References}

1. Haught JM, Patel S, English JC 3rd. Xanthoderma: a clinical review. J Am Acad Dermatol. 2007;57(6):1051-8.

2. Maharshak N, Shapiro J, Trau H. Carotenoderma-a review of the current literature. Int J Dermatol. 2003;42(3):178-81.

3. Lee YB, Lee KJ, Cho E, Cho BK, Park HJ. Carotenoderma in association with trastuzumab treatment. J Am Acad Dermatol. 2012;67(5):e201-2.

4. Dasanu CA, Alexandrescu DT, Dutcher J. Yellow skin discoloration associated with sorafenib use for treatment of metastatic renal cell carcinoma. South Med J. 2007;100(3):328-30.

5. Rietschel RL, Fowler JF. Fisher's contact dermatitis. Chapter 7, Noneczematous contact dermatitis. 6th edn. Ontario: BC Decker Inc; 2008. p 88-109.

6. $\mathrm{Xu} \mathrm{XB}$, Chen B, Liu WY. Curcumin inhibits the invasion of thyroid cancer cells via down-regulation of PI3K/Akt signaling pathway. Gene. 2014;546(2):226-32.

7. Committee on herbal medicinal products (HMPC) Assessment report on Curcuma longa L. rhizoma. 2009. http://www.ema. europa.eu/docs/en_GB/document_library/Herbal_-_HMPC_assessment_report/2010/02/WC500070700.pdf. Accessed 12 Nov 2009.

8. Prasad S, Tyagi AK, Aggarwal BB. Recent developments in delivery, bioavailability, absorption and metabolism of curcumin: the golden pigment from golden spice. Cancer Res Treat. 2014;46(1):2-18.

9. Pan MH, Huang TM, Lin JK. Biotransformation of curcumin through reduction and glucuronidation in mice. Drug Metab Dispos. 1999;27:486-94.

10. Nguyen TA, Friedman AJ. Curcumin: a novel treatment for skinrelated disorders. J Drugs Dermatol. 2013;12(10):1131-7.

11. Kanai M, Otsuka Y, Otsuka K, Sato M, Nishimura T, Mori Y, Kawaguchi M, Hatano E, Kodama Y, Matsumoto S, Murakami Y, Imaizumi A, Chiba T, Nishihira J, Shibata H. A phase I study investigating the safety and pharmacokinetics of highly bioavailable curcumin (Theracurmin) in cancer patients. Cancer Chemother Pharmacol. 2013;71(6):1521-30. 